\title{
Study of Fractional Analytic Functions and Local Fractional Calculus
}

\author{
Chii-Huei Yu
}

School of Mathematics and Statistics, Zhaoqing University, Guangdong Province, China

\begin{abstract}
Article Info

Volume 8, Issue 5

Page Number : 39-46

Publication Issue :

September-October-2021

In this present paper, the role of fractional analytic function in local fractional calculus is studied. Some important properties and theorems in local fractional calculus are discussed, such as product rule, quotient rule, chain rule, fundamental theorem of local fractional calculus, change of variable, integration by parts and so on. In addition, we propose several examples and formulas of local fractional calculus.
\end{abstract}

Article History

Accepted : 02 Sep 2021

Published: 07 Sep 2021
Keywords : Fractional Analytic Function, Local Fractional Calculus, Fundamental Theorem of Local Fractional Calculus, Product Rule, Quotient Rule, Chain Rule, Change of Variable, Integration by Parts.

\section{INTRODUCTION}

The importance of studying continuous but nowhere differentiable functions was emphasized a long time ago by Perrin, Poincare and others [1-2]. Local fractional calculus is a theory to study continuous non-differentiable functions and describe the mechanical behaviour in fractal space. It has developed for more than twenty years since Kolwankar and Gangal [3-4] used Riemann-Liouville definition of fractional derivative to obtain local fractional derivative operators. The local fractional derivative and integrals was broadly applied in the field of mathematics, applied science and engineering [5-16].

The purpose of the current paper is to study the role of fractional analytic functions in local fractional calculus. In Section II, we first give the definition of local fractional derivative, and we discuss some local fractional derivative properties, for example, product rule, Leibniz rule, quotient rule, chain rule and so on. Furthermore, we introduce some fractional functions and take them as examples. In Section III, the modified Riemann-Liouville fractional derivative of Jumarie type is introduced, and we provide several properties of this type of fractional derivative. In Section IV, we study fractional analytic functions and its role in local fractional derivative. In Section V, we give the definition of fractional Riemann integral (or called local fractional integral), and prove some important theorem such as fundamental theorem of local fractional calculus, change of variable, integration by parts. In Section VI, the conclusion is given. 


\section{LOCAL FRACTIONAL DERIVATIVE}

The purpose of this section is to state the definition and also introduce the properties of local fractional derivative.

Definition 2.1([16]): Let $0<\alpha<1,(-1)^{\alpha}=-1$, $f:[a, b] \rightarrow R$ and $x_{0} \in(a, b) . f$ is called local $\alpha-$ fractional differentiable at $x_{0}$ if $\lim _{x \rightarrow x_{0}} \frac{f(x)-f\left(x_{0}\right)}{\left(x-x_{0}\right)^{\alpha}}$ exists. And the $\alpha$-fractional derivative of $f(x)$ at $x_{0}$ is denoted by

$$
f^{(\alpha)}\left(x_{0}\right)=\Gamma(\alpha+1) \cdot \lim _{x \rightarrow x_{0}} \frac{f(x)-f\left(x_{0}\right)}{\left(x-x_{0}\right)^{\alpha}},
$$

where $\Gamma(\quad)$ is the gamma function. If $f$ is local $\alpha$ fractional differentiable at any point in open interval $(a, b)$, then we say that $f$ is a local $\alpha$-fractional differentiable function on $(a, b)$. In addition, the $n$-th order local $\alpha$-fractional derivative of $f(x)$ is denoted by $f^{(n \alpha)}(x)=\left(f^{(\alpha)}\right)\left(f^{(\alpha)}\right) \cdots\left(f^{(\alpha)}\right)(x)$, where $n$ is a positive integer, and $f^{(0)}(x)=f(x)$.

Theorem 2.2: Assume that $0<\alpha<1,(-1)^{\alpha}=-1$ and $x_{0} \in(a, b)$. If $f:[a, b] \rightarrow R$ is local $\alpha$-fractional differentiable at $x_{0}$, then $f$ is continuous at $x_{0}$.

$$
\begin{aligned}
& \text { Proof } \lim _{x \rightarrow x_{0}} f(x) \\
& =\lim _{x \rightarrow x_{0}}\left[f(x)-f\left(x_{0}\right)+f\left(x_{0}\right)\right] \\
& =\lim _{x \rightarrow x_{0}}\left[f(x)-f\left(x_{0}\right)\right]+f\left(x_{0}\right) \\
& \quad=\lim _{x \rightarrow x_{0}} \frac{f(x)-f\left(x_{0}\right)}{\left(x-x_{0}\right)^{\alpha}} \cdot \lim _{x \rightarrow x_{0}}\left(x-x_{0}\right)^{\alpha}+f\left(x_{0}\right) \\
& =\frac{f^{(\alpha)}\left(x_{0}\right)}{\Gamma(\alpha+1)} \cdot 0+f\left(x_{0}\right) \\
& =f\left(x_{0}\right) .
\end{aligned}
$$

Q.e.d.

Proposition 2.3: Let $0<\alpha<1,(-1)^{\alpha}=-1$, and $\lambda$ be a real number, If $f, g:[a, b] \rightarrow R$ are local $\alpha$-fractional differentiable at $x \in(a, b)$, then

(i) $\quad(f+g)^{(\alpha)}(x)=f^{(\alpha)}(x)+g^{(\alpha)}(x)$.

(ii) $\quad(f-g)^{(\alpha)}(x)=f^{(\alpha)}(x)-g^{(\alpha)}(x)$.

$$
\text { (iii) }
$$

$$
(\lambda f)^{(\alpha)}(x)=\lambda f^{(\alpha)}(x) \text {. }
$$

Proposition 2.4 (product rule for local fractional derivative):

$$
(f \cdot g)^{(\alpha)}(x)=f^{(\alpha)}(x) \cdot g(x)+f(x) \cdot g^{(\alpha)}(x)
$$

Proposition 2.5 (Leibniz rule for local fractional derivative):

$$
(f \cdot g)^{(n \alpha)}(x)=\sum_{k=0}^{n}\left(\begin{array}{l}
n \\
k
\end{array}\right) f^{((n-k) \alpha)}(x) \cdot g^{(k \alpha)}(x)
$$

Proposition 2.6 (quotient rule for local fractional derivative):

$$
\left(\frac{f}{g}\right)^{(\alpha)}(x)=\frac{f^{(\alpha)}(x) \cdot g(x)-f(x) \cdot g^{(\alpha)}(x)}{g^{2}} .
$$

In the following, some fractional functions are introduced.

Definition 2.7 ([22]): The Mittag-Leffler function is defined by

$$
E_{\alpha}(z)=\sum_{k=0}^{\infty} \frac{z^{k}}{\Gamma(k \alpha+1)},
$$

where $\alpha$ is a real number, $\alpha>0$, and $z$ is a complex variable.

Definition 2.8 ([17]): $E_{\alpha}\left(x^{\alpha}\right)$ is called $\alpha$-fractional exponential function, and the $\alpha$-fractional cosine and sine function are defined as follows:

$$
\cos _{\alpha}\left(x^{\alpha}\right)=\sum_{k=0}^{\infty} \frac{(-1)^{k} x^{2 k \alpha}}{\Gamma(2 k \alpha+1)},
$$

and

$$
\sin _{\alpha}\left(x^{\alpha}\right)=\sum_{k=0}^{\infty} \frac{(-1)^{k} x^{(2 k+1) \alpha}}{\Gamma((2 k+1) \alpha+1)}
$$

where $0<\alpha<1$, and $x$ is a real variable.

Example 2.9: We have the following local fractional derivative formulas:

$$
\begin{gathered}
E_{\alpha}\left(x^{\alpha}\right)^{(\alpha)}=E_{\alpha}\left(x^{\alpha}\right), \\
\sin _{\alpha}\left(x^{\alpha}\right)^{(\alpha)}=\cos _{\alpha}\left(x^{\alpha}\right),
\end{gathered}
$$

and

Therefore,

$$
\begin{gathered}
\left(\sin _{\alpha}\left(x^{\alpha}\right)+\cos _{\alpha}\left(x^{\alpha}\right)\right)^{(\alpha)}=\cos _{\alpha}\left(x^{\alpha}\right)-\sin _{\alpha}\left(x^{\alpha}\right), \\
\left(\sin _{\alpha}\left(x^{\alpha}\right)-\cos _{\alpha}\left(x^{\alpha}\right)\right)^{(\alpha)}=\cos _{\alpha}\left(x^{\alpha}\right)+\sin _{\alpha}\left(x^{\alpha}\right), \\
\left(\sin _{\alpha}\left(x^{\alpha}\right) \cdot \cos _{\alpha}\left(x^{\alpha}\right)\right)^{(\alpha)}=\left[\cos _{\alpha}\left(x^{\alpha}\right)\right]^{2}- \\
{\left[\sin _{\alpha}\left(x^{\alpha}\right)\right]^{2},}
\end{gathered}
$$$$
\cos _{\alpha}\left(x^{\alpha}\right)^{(\alpha)}=-\sin _{\alpha}\left(x^{\alpha}\right) .
$$ 


$$
\left(\frac{\sin _{\alpha}\left(x^{\alpha}\right)}{\cos _{\alpha}\left(x^{\alpha}\right)}\right)^{(\alpha)}=\frac{\left[\cos _{\alpha}\left(x^{\alpha}\right)\right]^{2}+\left[\sin _{\alpha}\left(x^{\alpha}\right)\right]^{2}}{\left[\cos _{\alpha}\left(x^{\alpha}\right)\right]^{2}} .
$$

We note that

$$
\left[\cos _{\alpha}\left(x^{\alpha}\right)\right]^{2}+\left[\sin _{\alpha}\left(x^{\alpha}\right)\right]^{2} \neq 1
$$

and

$$
\left[\cos _{\alpha}\left(x^{\alpha}\right)\right]^{2}-\left[\sin _{\alpha}\left(x^{\alpha}\right)\right]^{2} \neq \cos _{\alpha}\left(2 x^{\alpha}\right)
$$

for $0<\alpha<1$.

Remark 2.10: The followings are some local $\alpha$ fractional differentials.

$$
\begin{array}{r}
d_{\alpha} f=f^{(\alpha)} d x^{\alpha} . \\
d_{\alpha}(f+g)=d_{\alpha} f+d_{\alpha} g . \\
d_{\alpha}(f-g)=d_{\alpha} f-d_{\alpha} g . \\
d_{\alpha}(\lambda f)=\lambda d_{\alpha} f
\end{array}
$$

where $\lambda$ is a constant.

$$
\begin{gathered}
d_{\alpha}(f g)=d_{\alpha} f \cdot g+f \cdot d_{\alpha} g . \\
d_{\alpha}\left(\frac{f}{g}\right)=\frac{d_{\alpha} f \cdot g-f \cdot d_{\alpha} g}{g^{2}} .
\end{gathered}
$$

Proposition 2.11 (chain rule for local fractional derivative): Iff is $\alpha$-fractional differentiable at $u\left(x_{0}\right)$, and $u$ is differentiable at $x_{0}$, then $f(u(x))$ is $\alpha$ fractional differentiable at $x_{0}$ and

$$
(f \circ u)^{(\alpha)}\left(x_{0}\right)=f^{(\alpha)}\left(u\left(x_{0}\right)\right) \cdot\left(\frac{d u}{d x}\left(x_{0}\right)\right)^{\alpha} .
$$

Proof $(f \circ u)^{(\alpha)}\left(x_{0}\right)$

$=\lim _{x \rightarrow x_{0}} \frac{f(u(x))-f\left(u\left(x_{0}\right)\right)}{\left(x-x_{0}\right)^{\alpha}}$

$=\lim _{x \rightarrow x_{0}} \frac{f(u(x))-f\left(u\left(x_{0}\right)\right)}{\left(u(x)-u\left(x_{0}\right)\right)^{\alpha}} \cdot \frac{\left(u(x)-u\left(x_{0}\right)\right)^{\alpha}}{\left(x-x_{0}\right)^{\alpha}}$

$=\lim _{x \rightarrow x_{0}} \frac{f(u(x))-f\left(u\left(x_{0}\right)\right)}{\left(u(x)-u\left(x_{0}\right)\right)^{\alpha}} \cdot \lim _{x \rightarrow x_{0}}\left(\frac{u(x)-u\left(x_{0}\right)}{x-x_{0}}\right)^{\alpha}$

$=f^{(\alpha)}\left(u\left(x_{0}\right)\right) \cdot\left(\frac{d u}{d x}\left(x_{0}\right)\right)^{\alpha}$. $\quad$ Q.e.d.

\section{JUMARIE TYPE OF RIEMANN-LIOUVILLE FRACTIONAL DERIVATIVE}

In the following, we first give the definition of Jumarie type of fractional derivative.

Definition 3.1: Let $\alpha$ be a real number and $p$ be a positive integer. The modified Riemann-Liouville fractional derivative of Jumarie type ([17]) is defined by

$$
\left(x_{0} D_{x}^{\alpha}\right)[f(x)]=
$$

$$
\left\{\begin{array}{c}
\frac{1}{\Gamma(-\alpha)} \int_{x_{0}}^{x}(x-\tau)^{-\alpha-1} f(\tau) d \tau, \quad \text { if } \alpha<0 \\
\frac{1}{\Gamma(1-\alpha)} \frac{d}{d x} \int_{x_{0}}^{x}(x-\tau)^{-\alpha}[f(\tau)-f(a)] d \tau \text { if } 0 \leq \alpha<1 \\
\frac{d^{p}}{d x^{p}}\left(x_{0} D_{x}^{\alpha-p}\right)[f(x)], \quad \text { if } p \leq \alpha<p+1
\end{array}\right.
$$

In addition, if $\left(x_{0} D_{x}^{\alpha}\right)^{n}[f(x)]=\left({ }_{x_{0}} D_{x}^{\alpha}\right)\left({ }_{x_{0}} D_{x}^{\alpha}\right) \cdots$ $\left(x_{0} D_{x}^{\alpha}\right)[f(x)]$ exists, then $f(x)$ is called $n$-th order $\alpha$ fractional differentiable function, and $\left(x_{0} D_{x}^{\alpha}\right)^{n}[f(x)]$ is the $n$-th order $\alpha$-fractional derivative of $f(x)$. We note that $\left({ }_{x_{0}} D_{x}^{\alpha}\right)^{n} \neq{ }_{x_{0}} D_{x}^{n \alpha}$ in general. On the other hand, we define the $\alpha$ fractional integral of $f(x), \quad\left(x_{0} I_{x}^{\alpha}\right)[f(x)]=$ $\left(x_{0} D_{x}^{-\alpha}\right)[f(x)]$, where $\alpha>0$. And $f(x)$ is called $\alpha$ -fractional integrable function. We have the following property.

Proposition 3.2 ([18]): Let $\alpha, \beta, c$ be real numbers and $\beta \geq \alpha>0$, then

$$
\begin{gathered}
\left({ }_{0} D_{x}^{\alpha}\right)\left[x^{\beta}\right]=\frac{\Gamma(\beta+1)}{\Gamma(\beta-\alpha+1)} x^{\beta-\alpha}, \\
{ }_{0} D_{x}^{\alpha}[c]=0 \\
\left({ }_{0} D_{x}^{\alpha}\right)\left[E_{\alpha}\left(x^{\alpha}\right)\right]=E_{\alpha}\left(x^{\alpha}\right) . \\
\left({ }_{0} D_{x}^{\alpha}\right)\left[\sin _{\alpha}\left(x^{\alpha}\right)\right]=\cos _{\alpha}\left(x^{\alpha}\right) . \\
\left({ }_{0} D_{x}^{\alpha}\right)\left[\cos _{\alpha}\left(x^{\alpha}\right)\right]=-\sin _{\alpha}\left(x^{\alpha}\right) .
\end{gathered}
$$

\section{FRACTIONAL ANALYTIC FUNCTIONS}

Definition 4.1: Assume that $x, x_{0}$ and $a_{n}$ are real numbers, $x_{0} \in(a, b)$, and $0<\alpha<1$. If the function $f:[a, b] \rightarrow R$ can be expressed as a $\alpha$-fractional power series, that is, $f(x)=\sum_{n=0}^{\infty} a_{n}\left(x-x_{0}\right)^{n \alpha}$ on some open interval $\left(x_{0}-r, x_{0}+r\right)$, then we say that $f(x)$ is $\alpha$-fractional analytic at $x_{0}$, where $r$ is the radius of convergence about $x_{0}$. If $f:[a, b] \rightarrow R$ is continuous on closed interval $[a, b]$ and is $\alpha$-fractional analytic at every point in open interval $(a, b)$, then $f$ is called a $\alpha$-fractional analytic function on $[a, b]$.

Proposition 4.2: Assume that $0<\alpha<1,(-1)^{\alpha}=$ $-1, a<b, p \neq 0, c \in(a, b)$, and let $f(x)=(x-c)^{p}$ defined on $[a, b]$. 
Case 1. If $p \neq \alpha$, then $f(x)$ is not $\alpha$-fractional analytic at all points in $(a, b)$.

Case 2. If $p=\alpha$, then $f(x)$ is $\alpha$-fractional analytic only at $x=c$.

Proof If $p>\alpha$. Since $f^{(\alpha)}(x)=0$ for all $x \in(a, b)$. It follows that for any positive integer $n, f^{(n \alpha)}(x)=0$ for all $x \in(a, b)$. And hence, $f(x)$ is not $\alpha$-fractional analytic at all points in $(a, b)$. Since if $(x)$ is $\alpha$ fractional analytic at some point $\eta \in(a, b)$, then $f(x)$ is a constant function, which is a contradiction.

If $p<\alpha$. We have

$$
f^{(\alpha)}(x)=\left\{\begin{array}{cc}
0 & \text { if } x \neq c \\
\text { nonexist } \text { if } x=c
\end{array}\right.
$$

Thus,

$$
f^{(n \alpha)}(x)=\left\{\begin{array}{cc}
0 & \text { if } x \neq c \\
\text { nonexist } \text { if } x=c .
\end{array}\right.
$$

for all positive integers $n$ and all $x \in(a, b)$.

And hence, $f(x)$ is not $\alpha$-fractional analytic at all points in $(a, b)$.

If $p=\alpha$. Then

$$
f^{(\alpha)}(x)=\left\{\begin{array}{c}
0 \quad \text { if } x \neq c \\
\Gamma(\alpha+1) \text { if } x=c .
\end{array}\right.
$$

Therefore, $f^{(n \alpha)}(x)=0$ for all $n \geq 2$ and all $x \in$ $(a, b)$. So, $f(x)$ is $\alpha$-fractional analytic only at $x=c$.

Q.e.d.

Theorem 4.3: Suppose that $0<\alpha<1$ and $(-1)^{\alpha}=$ -1 . If $f:[a, b] \rightarrow R$ is $\alpha$-fractional analytic at $x_{0} \in$ $(a, b)$, then

$$
f^{(\alpha)}\left(x_{0}\right)=\left(x_{0} D_{t}^{\alpha}\right)[f(t)]\left(x_{0}\right) .
$$

Proof By Theorem 2.9 in [19], we can immediately obtain this result.

Q.e.d.

Corollary 4.4: Let $0<\alpha<1$ and $(-1)^{\alpha}=-1$. If $f:[a, b] \rightarrow R$ is $\alpha$-fractional analytic on $[a, b]$, then

$$
f^{(n)}(x)=\left({ }_{x} D_{t}^{\alpha}\right)^{n}[f(t)](x)
$$

for all positive integers $n$ and all points $x \in(a, b)$.

Proof Using Theorem 4.3 and by induction, we can easily obtain the result.

Q.e.d.

Theorem 4.5: Let $0<\alpha<1$. If $f(x)=\sum_{n=0}^{\infty} a_{n}(x-$ $\left.x_{0}\right)^{n \alpha}$, then

$$
f(x)=\sum_{n=0}^{\infty} \frac{f^{(n \alpha)}\left(x_{0}\right)}{\Gamma(n \alpha+1)}\left(x-x_{0}\right)^{n \alpha} .
$$

Proof By Theorem 2.11 in [20] and the above Corollary 4.4, the desired result holds.

Q.e.d.

Theorem 4.6 (mean value theorem for local fractional derivative): Suppose that $0<\alpha<1$ and $(-1)^{\alpha}=-1$. If $f$ is $\alpha$-fractional analytic on $[a, b]$, then there exists $\xi \in(a, b)$ such that

$$
f(b)-f(a)=\frac{f^{(\alpha)}(\xi)}{\Gamma(\alpha+1)}(b-a)^{\alpha} .
$$

Proof Using Theorem 2.12 in [19] and the above Theorem 4.3, we obtain the desired result. Q.e.d.

Theorem 4.7: Let $0<\alpha<1$ and $(-1)^{\alpha}=-1$. If $f$ is $\alpha$-fractional analytic on $[a, b]$ and $f^{(\alpha)}(x)=0$ for all $x \in(a, b)$, then $f$ is a constant function on $[a, b]$.

Proof Using mean value theorem for local fractional derivative yields $f(x)=f(a)$ for all $x \in[a, b]$. And hence, the desired result holds. $\quad$ Q.e.d.

\section{FRACTIONAL RIEMANN INTEGRAL}

Definition 5.1: Let $0<\alpha<1$, and $f:[a, b] \rightarrow R$. If

$$
\lim _{\|\Delta\| \rightarrow 0} \sum_{k=1}^{n} f\left(\xi_{k}\right)\left(x_{k}-x_{k-1}\right)^{\alpha}
$$

exists, where the partitions of the interval $[a, b]$ are denoted by $\left[x_{k-1}, x_{k}\right], k=1, \cdots, n, x_{0}=a, x_{n}=b$, $\xi_{k} \in\left[x_{k-1}, x_{k}\right] \quad, \quad \Delta x_{k}=x_{k}-x_{k-1}, \quad$ and $\|\Delta\|=$ $\max _{k=1, \cdots, n}\left\{\Delta x_{k}\right\}$. Then we say $f$ is a $\alpha$-fractional Riemann integrable function on $[a, b]$. And we denoted as

$$
\lim _{\|\Delta\| \rightarrow 0} \sum_{k=1}^{n} f\left(\xi_{k}\right)\left(x_{k}-x_{k-1}\right)^{\alpha}=\int_{a}^{b} f(x) d x^{\alpha},
$$

which is called the $\alpha$-fractional Riemann integral (or local $\alpha$-fractional integral) of $f$ on $[a, b]$.

Proposition 5.2: Assume that $0<\alpha<1$ and $\lambda$ is a real number. If $f, g:[a, b] \rightarrow R$ are $\alpha$-fractional Riemann integrable functions on $[a, b]$, then

(i) $\int_{a}^{b}[f(x)+g(x)] d x^{\alpha}=\int_{a}^{b} f(x) d x^{\alpha}+\int_{a}^{b} g(x) d x^{\alpha}$. 
(ii) $\int_{a}^{b}[f(x)-g(x)] d x^{\alpha}=\int_{a}^{b} f(x) d x^{\alpha}-$ $\int_{a}^{b} g(x) d x^{\alpha}$.

$$
\int_{a}^{b} \lambda f(x) d x^{\alpha}=\lambda \int_{a}^{b} f(x) d x^{\alpha}
$$

(iv) If $c \in(a, b)$, then

$$
\int_{a}^{c} f(x) d x^{\alpha}+\int_{c}^{b} f(x) d x^{\alpha}=\int_{a}^{b} f(x) d x^{\alpha} .
$$

(v) Iff $\geq 0$, then $\int_{a}^{b} f(x) d x^{\alpha} \geq 0$.

(vi) Iff $\leq g$, then $\int_{a}^{b} f(x) d x^{\alpha} \leq \int_{a}^{b} g(x) d x^{\alpha}$.

Proposition 5.3: If $f$ is $\alpha$-fractional Riemann integrable on $[a, b]$, then $f$ is bounded on $[a, b]$.

Proof If $f$ is not bounded on $[a, b]$, then for any partition $P$ of $[a, b]$, the function $f$ is not bounded on some interval $\left[x_{k-1}, x_{k}\right]$ of $P$. By choosing the point $\xi_{k} \in\left[x_{k-1}, x_{k}\right]$ in different ways, we can make the quantity $\left|f\left(\xi_{k}\right)\left(x_{k}-x_{k-1}\right)^{\alpha}\right|$ as large as desired. Thus, the $\alpha$-fractional Riemann sum $\sum_{k=1}^{n} f\left(\xi_{k}\right)\left(x_{k}-\right.$ $\left.x_{k-1}\right)^{\alpha}$ can also be made as large as desired in absolute value by changing only the point $\xi_{k}$ in this interval. That is, $f$ is not $\alpha$-fractional Riemann integrable on $[a, b]$.

Q.e.d.

Theorem 5.4 (fundamental theorem of local fractional calculus): Let $0<\alpha<1,(-1)^{\alpha}=-1$. If $F:[a, b] \rightarrow$ $R$ satisfies $F^{(\alpha)}(x)=f(x)$ for all $x \in(a, b)$, then

$$
\int_{a}^{b} f(x) d x^{\alpha}=\Gamma(\alpha+1)(F(b)-F(a)) .
$$

Proof Let $\left\{\left[x_{k-1}, x_{k}\right]\right\}_{k=1, \ldots, n}$ be any partition of the interval $[a, b]$, then by mean value theorem for local fractional derivative and the definition of fractional Riemann integral, we obtain

$\Gamma(\alpha+1)(F(b)-F(a))$

$=\Gamma(\alpha+1)\left[\left(F\left(x_{0}\right)-F\left(x_{1}\right)\right)+\left(F\left(x_{1}\right)-F\left(x_{2}\right)\right)+\cdots\right.$ $+\left(F\left(x_{n-1}\right)-F\left(x_{n}\right)\right)$

$=\Gamma(\alpha+1)\left[\left(\frac{F\left(x_{0}\right)-F\left(x_{1}\right)}{\left(x_{0}-x_{1}\right)^{\alpha}} \cdot\left(x_{0}-x_{1}\right)^{\alpha}\right)\right.$

$+\left(\frac{F\left(x_{1}\right)-F\left(x_{2}\right)}{\left(x_{1}-x_{2}\right)^{\alpha}} \cdot\left(x_{1}-x_{2}\right)^{\alpha}\right)+\cdots$

$\left.+\left(\frac{F\left(x_{n-1}\right)-F\left(x_{n}\right)}{\left(x_{n-1}-x_{n}\right)^{\alpha}} \cdot\left(x_{n-1}-x_{n}\right)^{\alpha}\right)\right]$

$=\left[\left(f^{(\alpha)}\left(\xi_{1}\right) \cdot\left(x_{0}-x_{1}\right)^{\alpha}\right)+\left(f^{(\alpha)}\left(\xi_{2}\right) \cdot\left(x_{1}-x_{2}\right)^{\alpha}\right)\right.$

$$
\begin{array}{rr} 
& \left.+\cdots+\left(f^{(\alpha)}\left(\xi_{n}\right) \cdot\left(x_{n-1}-x_{n}\right)^{\alpha}\right)\right] \\
= & \int_{a}^{b} f(x) d x^{\alpha} .
\end{array}
$$

Remark 5.5: If $f:[a, b] \rightarrow R$ is a $\alpha$-fractional analytic function on $[a, b]$, then by fundamental theorem of local fractional calculus, we have

$$
\frac{d}{d x^{\alpha}} \int_{a}^{x} f(t) d t^{\alpha}=\Gamma(\alpha+1) f(x)
$$

for all $x \in(a, b)$.

On the other hand, if $F:[a, b] \rightarrow R$ is $\alpha$-fractional analytic on $[a, b]$, then

$$
\int_{a}^{b} F^{(\alpha)}(x) d x^{\alpha}=\Gamma(\alpha+1)(F(b)-F(a)) .
$$

Remark 5.6: Fundamental theorem of local fractional calculus cannot hold for non-fractional analytic functions. For example, by Proposition 4.2, we know that $F(x)=x^{2}$ defined on $[0,1]$, is not $\frac{1}{2}$-fractional analytic function at all points in $[0,1]$. And by the definition of local fractional derivative, we know that $F^{(1 / 2)}(x)=0$ for all points $x \in[0,1]$. Therefore,

$$
\int_{0}^{1} F^{(1 / 2)}(x) d x^{1 / 2} \neq \Gamma\left(\frac{3}{2}\right) \cdot(F(1)-F(0)) .
$$

Theorem 5.7: Suppose that $f$ is a $\alpha$-fractional analytic function, and $u(x), w(x)$ are differentiable functions, then

$$
\begin{aligned}
& \frac{d}{d x^{\alpha}} \int_{w(x)}^{u(x)} f(t) d t^{\alpha} \\
= & f(u(x)) \cdot\left(\frac{d u}{d x}\right)^{\alpha}-f(w(x)) \cdot\left(\frac{d w}{d x}\right)^{\alpha} .
\end{aligned}
$$

Proof Taking a point $c$ contained in the domain of $f$. Let $F(x)=\int_{a}^{x} f(t) d t^{\alpha}$, then

$$
F(u(x))=\int_{a}^{u(x)} f(t) d t^{\alpha},
$$

and

$$
F(w(x))=\int_{a}^{w(x)} f(t) d t^{\alpha}
$$

Thus,

$$
\int_{w(x)}^{u(x)} f(t) d t^{\alpha}=F(u(x))-F(w(x)) .
$$

Therefore, using chain rule for local fractional derivative yields

$\frac{d}{d x^{\alpha}} \int_{w(x)}^{u(x)} f(t) d t^{\alpha}$ 


$$
\begin{aligned}
& =\frac{d}{d x^{\alpha}} F(u(x))-\frac{d}{d x^{\alpha}} F(w(x)) \\
& =F^{(\alpha)}(u(x)) \cdot\left(\frac{d u}{d x}(x)\right)^{\alpha}-F^{(\alpha)}(w(x)) \cdot\left(\frac{d w}{d x}(x)\right)^{\alpha} \\
& =f(u(x)) \cdot\left(\frac{d u}{d x}(x)\right)^{\alpha}-f(w(x)) \cdot\left(\frac{d w}{d x}(x)\right)^{\alpha} .
\end{aligned}
$$

Q.e.d.

Theorem 5.8 (change of variable for local fractional integral): Suppose that $0<\alpha<1,(-1)^{\alpha}=-1$, $f:[a, b] \rightarrow R$ is a $\alpha$-fractional analytic function, and $u:[c, d] \rightarrow[a, b]$ is a differentiable function with $u(c)=a, u(d)=b$. If $f(u(x))\left(\frac{d u}{d x}\right)^{\alpha}$ is $\alpha$-fractional analytic on $[c, d]$, then

$$
\int_{c}^{d} f(u(x))\left(\frac{d u}{d x}\right)^{\alpha} d x^{\alpha}=\int_{a}^{b} f(u) d u^{\alpha} .
$$

Proof Fix $x_{0} \in[a, b]$ and let $F(u)=\int_{x_{0}}^{u} f(t) d t^{\alpha}$. Since $f$ is $\alpha$-fractional analytic on $[a, b]$, it follows from fundamental theorem of local fractional calculus that $F^{(\alpha)}(u)=f(u)$ for all $u \in[a, b]$. Let $g=F \circ u$. Since $f(u(x))\left(\frac{d u}{d x}\right)^{\alpha}$ is $\alpha$-fractional analytic on $[c, d]$, using chain rule for local fractional derivative yields

$$
g^{(\alpha)}(x)=F^{(\alpha)}(u(x))\left(\frac{d u}{d x}\right)^{\alpha}=f(u(x))\left(\frac{d u}{d x}\right)^{\alpha} .
$$

And hence $g$ is analytic on $[c, d]$. Furthermore,

$$
\begin{aligned}
& \int_{c}^{d} f(u(x))\left(\frac{d u}{d x}\right)^{\alpha} d x^{\alpha} \\
= & \int_{c}^{d} g^{(\alpha)}(x) d x^{\alpha} \\
= & g(d)-g(c) \\
= & F(u(d))-F(u(c)) \\
= & \int_{x_{0}}^{u(d)} f(t) d t^{\alpha}-\int_{x_{0}}^{u(c)} f(t) d t^{\alpha} \\
= & \int_{u(c)}^{u(d)} f(t) d t^{\alpha}
\end{aligned}
$$$$
=\int_{a}^{b} f(u) d u^{\alpha} .
$$

Q.e.d.
Theorem 5.9 (integration by parts for local fractional calculus): If $f, g$ are $\alpha$-fractional analytic functions on $[a, b]$, then

$$
\begin{aligned}
& \int_{a}^{b} f(x) \cdot g^{(\alpha)}(x) d x^{\alpha} \\
& \quad=\left.f(x) \cdot g(x)\right|_{a} ^{b}-\int_{a}^{b} g(x) \cdot f^{(\alpha)}(x) d x^{\alpha} .
\end{aligned}
$$

Proof Since by product rule for local fractional derivative, we have

$$
f(x) \cdot g^{(\alpha)}(x)=(f \cdot g)^{(\alpha)}(x)-f^{(\alpha)}(x) \cdot g(x) .
$$

It follows from fundamental theorem of local fractional calculus that

$$
\begin{aligned}
& \int_{a}^{b} f(x) \cdot g^{(\alpha)}(x) d x^{\alpha} \\
& =\int_{a}^{b}(f \cdot g)^{(\alpha)}(x) d x^{\alpha}-\int_{a}^{b} f^{(\alpha)}(x) \cdot g(x) d x^{\alpha} \\
& =\left.f(x) \cdot g(x)\right|_{a} ^{b}-\int_{a}^{b} g(x) \cdot f^{(\alpha)}(x) d x^{\alpha} .
\end{aligned}
$$

Q.e.d.

The followings are some indefinite local fractional integral formulas.

Proposition 5.10: Let $0<\alpha<1$ and $C$ be a constant. Then

$$
\begin{gathered}
\int E_{\alpha}\left(x^{\alpha}\right) d x^{\alpha}=\Gamma(\alpha+1) \cdot E_{\alpha}\left(x^{\alpha}\right)+C, \\
\int \sin _{\alpha}\left(x^{\alpha}\right) d x^{\alpha}=-\Gamma(\alpha+1) \cdot \cos _{\alpha}\left(x^{\alpha}\right)+C, \\
\int \cos _{\alpha}\left(x^{\alpha}\right) d x^{\alpha}=\Gamma(\alpha+1) \cdot \sin _{\alpha}\left(x^{\alpha}\right)+C, \\
\int \sum_{k=0}^{\infty} \frac{a_{n}}{\Gamma(k \alpha+1)} x^{k \alpha} d x^{\alpha} \\
=\Gamma(\alpha+1) \cdot \sum_{k=0}^{\infty} \frac{a_{n}}{\Gamma((k+1) \alpha+1)} x^{(k+1) \alpha}+C,
\end{gathered}
$$

where $\sum_{k=0}^{\infty} \frac{a_{n}}{\Gamma(k \alpha+1)} x^{k \alpha}$ is a $\alpha$-fractional analytic function on some closed interval.

Remark 5.11: We note that the formulas

$$
\int 1 d x^{\alpha}=x^{\alpha}+C
$$

and

$$
\int x^{k \alpha} d x^{\alpha}=\frac{\Gamma(k \alpha+1) \cdot \Gamma(\alpha+1)}{\Gamma((k+1) \alpha+1)} \cdot x^{(k+1) \alpha}+C
$$

are false ( $k$ is any positive integer). Since by Proposition 4.2, the local $\alpha$-fractional derivatives of the functions $F(x)=x^{\alpha}+C$ and $G(x)=$ $\frac{\Gamma(k \alpha+1) \cdot \Gamma(\alpha+1)}{\Gamma((k+1) \alpha+1)} \cdot x^{(k+1) \alpha}+C, \quad$ are $\quad F^{(\alpha)}(x)=0$ and $G^{(\alpha)}(x)=0$ for all $x \neq 0$. 
From the above discussion, we know that the fractional analytic function plays an important role in local fractional calculus. And the Jumarie type of fractional calculus is equivalent to local fractional calculus when the studied function is fractional analytic. As well, we discussed some important properties in local fractional calculus, for example, fractional mean value theorem, fundamental theorem of local fractional calculus, fractional integration by parts, fractional change of variable and so on. In fact, these theorems are natural generalizations of the ones in classical calculus. In the future, the results in local fractional calculus we obtained will be used to extend the research topics to applied science and engineering mathematics.

\section{REFERENCES}

[1]. T. W. Korner, Fourier Analysis, Cambridge University Press, Cambridge, 1989.

[2]. B. B. Mandelbrot, The Fractal Geometry of Nature, Freeman, New York, 1977.

[3]. K. M. Kolwanka, A. D. Gangal, "Fractional differentiability of nowhere differentiable functions and dimensions," Journal of Chaos, Vol. 6, pp. 505-513, 1996.

[4]. K. M. Kolwanka, A. D. Gangal, "Holder exponents of irregular signals and local fractional deirvatives, " PRAMANA-Journal of Physics, Vol. 48, No. 1, pp.49-68, 1997.

[5]. X. -J. Yang, C. Cattani, G. Xie, "Local fractional calculus application to differential equations arising in fractal heat transfer," Fractional Dynamics, pp.282-295, 2015.

[6]. A. Carpinteri, P. Cornetti, "A fractional calculus approach to the description of stress and strain localization in fractal media," Chaos, Solitons and Fractals, Vol. 13, pp. 85-94, 2002.

[7]. A. -M. Yang, Y. -Z. Zhang, C. Cattani et al., "Application of local fractional series expansion method to solve Klein-Gordon equations on
Cantor sets," Abstract and Applied Analysis, Vol. 2014, Article ID 372741, 6 pages, 2014.

[8]. X. -J. Yang, Advanced Local Fractional Calculus and Its Applications, World Science Publisher, New York, NY, USA, 2012.

[9]. J. H. He, "Local fractional variational iteration method for fractal heat transfer in silk cocoon hierarchy," Nonlinear Science Letters A, Vol. 4, No. 1, pp. 15-20, 2013.

[10]. X. -J. Yang and D. Baleanu, "Local fractional variational iteration method for Fokker-Planck equation on a Cantor set," Acta Universitaria, Vol. 23, No. 2, pp. 3-8, 2013.

[11]. C. -G. Zhao, A. -M. Yang, H. Jafari, and A. Haghbin, "The Yang-Laplace transform for solving the IVPs with local fractional derivative," Abstract and Applied Analysis, Vol. 2014, Article ID 386459, 5 pages, 2014.

[12]. E. Lutz, "Fractional transport equations for Levy stable processes," Physical Review Letters, Vol. 86, No. 11, pp. 2208-2211, 2001.

[13]. Y. Zhao, D. Baleanu, C. Cattani, et al., "Maxwell's equations on Cantor sets: a local fractional approach," Advances in High Energy Physics, Article ID686371, 6pages, 2013.

[14]. A. M. Yang, C. Cattani, Zhang, et al., "Local fractional Fourier series solutions for nonhomogeneous heat equations arising in fractal heat flow with local fractional derivative," Advances in Mechanical Engineering, 2014, Article ID514639, 5pages, 2014.

[15]. X. -J. Yang, D. Baleanu, "Fractal heat conduction problem solved by local fractional variation iteration method," Thermal Science, Vo. 17, No. 2, pp. 625-628, 2013.

[16]. M. Li, X. -F. Hui, C. Cattani, X. -J. Yang, and Y. Zhao, "Approximate Solutions for Local Fractional Linear Transport Equations Arising in Fractal Porous Media," Advances in Mathematical Physics, Vol. 2014, pp. 1-8, 2014. 
[17]. C. - $\mathrm{H} . \mathrm{Yu}$, "A new insight into fractional logistic equation," International Journal of Engineering Research and Reviews, Vol. 9, Issue 2, pp.13-17, 2021.

[18]. U. Ghosh, S. Sengupta, S. Sarkar, and S. Das, "Analytic solution of linear fractional differential equation with Jumarie derivative in term of Mittag-Leffler function," American Journal of Mathematical Analysis, Vol. 3, No. 2, pp.32-38, 2015.

[19]. C. -H. Yu, "Fractional mean value theorem and its applications," International Journal of Electrical and Electronics Research, Vol. 9, Issue 2, pp. 19-24, 2021.

[20]. C. - H. Yu, "Fractional Taylor series based on Jumarie type of modified Riemann-Liouville derivatives," International Journal of Latest Research in Engineering and Technology, Vol. 7, Issue 6, pp.1-6, 2021.

\section{Cite this article as :}

Chii-Huei Yu, "Study of Fractional Analytic Functions and Local Fractional Calculus", International Journal of Scientific Research in Science, Engineering and Technology (IJSRSET), Online ISSN : 2394-4099, Print ISSN : 2395-1990, Volume 8 Issue 5, pp. 39-46, September-October 2021. Available at doi : https://doi.org/10.32628/IJSRSET218482 Journal URL : https://ijsrset.com/IJSRSET218482 